\title{
Bowel Preparation for Surveillance Colonoscopy After Colorectal Resection: A New Perspective
}

\author{
Donghyoun Lee ${ }^{1,2}$, Ho-Kyung Chun ${ }^{2}$ \\ ${ }^{1}$ Department of Surgery, Jeju National University Hospital, Jeju National University School of Medicine, Jeju; ${ }^{2}$ Department of Surgery, \\ Kangbuk Samsung Hospital, Sungkyunkwan University School of Medicine, Seoul, Korea
}

Purpose: Inadequate bowel preparation (IBP) is commonly observed during surveillance colonoscopy after colorectal resection. We investigated potential risk factors affecting bowel preparation.

Methods: We studied potential factors affecting bowel preparation quality. The Boston bowel preparation score was used to measure bowel preparation quality. Factors affecting IBP were analyzed, including age, body mass index, time elapsed between surgery and colonoscopy, and amount of bowel preparation drug consumed (conventional-volume vs. low-volume). Odds ratios were calculated for IBP.

Results: This retrospective cohort study included 1,317 patients who underwent colorectal resection due to malignancy. Of these patients, $79 \%$ had adequate bowel preparation and $21 \%$ had IBP. In multivariate regression analysis, a surveillance colonoscopy within 1 year after surgery and age $>80$ were used as independent predictors of IBP. IBP rate of the lowvolume group was significantly higher than that of the conventional-volume group among patients who underwent a surveillance colonoscopy within 1 year after surgery.

Conclusion: For surveillance colonoscopy after colorectal resection, bowel preparation is affected by factors including colonoscopy timing after surgery and age. We recommend the use of conventional-volume 4-L polyethylene glycol solution when performing a surveillance colonoscopy, especially up to 1 year after surgery.

\section{Keywords: Colonoscopy; Colonic neoplasms; Rectal neoplasms}

\section{INTRODUCTION}

Colorectal cancer (CRC) is the fourth leading cause of cancer-related death in South Koreans, and the third leading cause of death worldwide [1]. Because of its prevalence, surgical techniques and systemic therapies have advanced over the past 20 years, and are correlated with an increase in postoperative CRC survival rate throughout most of the world (with exceptions for patients in Eastern Europe and Latin America) [2]. As a result, the number

Received: October 17, 2018 - Accepted: November 8, 2018

Correspondence to: Ho-Kyung Chun, M.D.

Department of Surgery, Kangbuk Samsung Hospital, Sungkyunkwan University School of Medicine, 29 Saemunan-ro, Jongno-gu, Seoul 03181, Korea

Tel: +82-2-2001-1300, Fax: +82-2-2001-2131, E-mail: hkchun@skku.edu ORCID code: https://orcid.org/0000-0001-6447-3550

(C) 2019 The Korean Society of Coloproctology

This is an open-access article distributed under the terms of the Creative Commons Attribution NonCommercial License (http://creativecommons.org/licenses/by-nc/4.0) which permits unrestricted noncommercial use, distribution, and reproduction in any medium, provided the original work is properly cited. of patients who underwent surveillance after colorectal resection has increased. Although no standard for surveillance has been established, it is common to combine some or all of the following techniques for effective surveillance, including physical examinations, blood carcinoembryonic antigen tests, computed tomography scans of the chest and abdomen, and endoscopy. Existing studies have shown that patients that undergo a colonoscopy after CRC resection have a lower overall mortality rate and a lower risk of metachronous cancer during the perioperative period compared to those who do not undergo colonoscopy [3]. As a result, colonoscopy has become increasingly more important as a surveillance method for the diagnostic evaluation of metachronous cancer and its recurrence.

Recently, ways to increase surveillance colonoscopy effectiveness for early diagnosis of CRC recurrence or metachronous cancer have been investigated, with many studies focusing on the ideal time period between surgery and surveillance colonoscopy. The current incidence rate of metachronous colon cancer ranges from $0.7 \%$ to $3.6 \%$ within 3 years postsurgery, and $90 \%$ of cancer recur- 
rence is observed within 5 years of surgery [4]. Based on these findings, several studies recommend that patients who undergo $\mathrm{CRC}$ resection should undergo a first surveillance colonoscopy one year after surgery [5]. Furthermore, the National Comprehensive Cancer Network guidelines recommend postoperative surveillance colonoscopy within the first year after CRC surgery [6].

A critical prerequisite for surveillance colonoscopy is that the examination must be effective and of high-quality. A high-quality colonoscopy assumes completeness (where the cecum or anastomosis is reached), adequate bowel preparation, and meticulous examination by appropriately trained operators who meet adenoma detection benchmarks (i.e., frequency of conventional adenoma detection $>25 \%$ in average-risk screening colonoscopies) [7]. In particular, of these prerequisites, adequate bowel preparation with no solid or liquid fecal material in the bowel mucosa plays a critical role in tumor detection. Previous studies have shown that inadequate bowel preparation (IBP) at the time of $\mathrm{CRC}$ screening colonoscopies resulted in an adenoma miss rate ranging from $33 \%$ to $46 \%$ [8]. In addition, adequate bowel preparation is essential not only for increased detection of metachronous cancers or lesions, but also to avoid unfavorable results such as cecal intubation failure or prolonged procedural time [9].

A large amount of liquid fecal material was often found in the colon when surveillance colonoscopies were performed on patients who underwent colorectal resection, despite the fact that patients had been educated on bowel preparation and reported intake of all bowel preparation medications. Nonevacuated bowels may occur for various reasons, including inability to completely evacuate the bowel due to decreased bowel motility after surgery and reduced compliance of bowel preparation because of repeated tests. Accurately quantifying and assessing reasons for poor bowel preparation is difficult; as such, few studies on bowel preparation for surveillance colonoscopy have been performed compared to colonoscopy studies focused on histopathologic results or ideal time period between colonoscopies. Bowel preparation for surveillance colonoscopies has received relatively little attention.

In this study, we sought to determine the risk factors for IBP surveillance colonoscopy after colorectal resection.

\section{METHODS}

\section{Study population}

This study was approved by the Institutional Review Board at Kangbuk Samsung Hospital and was conducted according to the Declaration of Helsinki (KBSMC 2017-02-012). The informed consent of patients was waived because of the retrospective nature of the study. The medical records of 1,393 patients who underwent elective colorectal resection for malignancy were included in this study. All operations were performed by consultant-level surgeons in the hospital. All examinations were performed by a single colonoscopist (HOK) who has extensive experience perform- ing over 10,000 colonoscopy procedures. Bowel adhesion development is the most common sequelae of intra-abdominal and pelvic surgery [10]; therefore, we excluded patients who underwent open abdominal surgery. Exclusion criteria included (1) patients who underwent open abdominal colectomy, (2) patients who underwent other abdominal surgery during or after colorectal resection, (3) patients who underwent anastomosis through a method other than end-to-end anastomosis, and (4) patients who had conditions such as stricture or obstruction that can cause intraluminal passage after colorectal resection. Systemic treatment was defined to include both adjuvant and neoadjuvant chemotherapy.

\section{Bowel preparation method}

The bowel preparation method was determined by the surgeon. The chosen method was either a low-volume combination of 2 bottles of PICO (10-mg sodium picosulfate, 3.5-g magnesium oxide, and 12-g citric acid; Picolight, Pharmbio, Seoul, Korea) combined with Magcorol solution (19.5-g citric acid and 10.75-g magnesium carbonate; Taejoon Pharm. Co., Seoul, Korea), or a conventional-volume 4-L polyethylene glycol (PEG) solution (CoLyte and CoLyte-F, Taejoon Pharm. Co.; Colonlyte, Dream Pharma, Seoul, Korea). All patients scheduled for a colonoscopy in the morning followed a soft-blended or fluid diet for breakfast and lunch and then were allowed only water for the remainder of the day before the colonoscopy. Patients scheduled for a colonoscopy in the afternoon were allowed breakfast, lunch, and dinner before 5:00 PM and water for the remainder of the day before the colonoscopy. All patients in the low-volume group were asked to consume one bottle of PICO solution at 5:00 PM the day before the colonoscopy, followed by another bottle of PICO solution with 1 $\mathrm{L}$ of water at 8:00 PM. In the conventional-volume group, patients scheduled for a colonoscopy in the morning were asked to consume $2 \mathrm{~L}$ of Coolprep with $500 \mathrm{~mL}$ of water at 9:00 PM the day before the colonoscopy, and they were given a second $2 \mathrm{~L}$ of Coolprep with $500 \mathrm{~mL}$ of water at 5:00 AM on the day of the colonoscopy. Patients scheduled for a colonoscopy in the afternoon were asked to consume $2 \mathrm{~L}$ of Coolprep with $500 \mathrm{~mL}$ of water at 6:00 AM the day of the colonoscopy and then a second $2 \mathrm{~L}$ of Coolprep with $1 \mathrm{~L}$ of water at 8:30 $\mathrm{AM}$ on the day of the colonoscopy. These bowel preparation methods were explained to the patients by well-trained nurses. Before each colonoscopy, face-to-face interviews were performed, and the colonoscopy was postponed if the patient reported an insufficient intake of the bowel preparation drug and defecation was not limited to clear fluid.

\section{Colonoscopic examination}

Surveillance colonoscopy was defined as a colonoscopy performed to monitor patients who underwent left-sided colectomy in response to CRC. Benign anastomotic stricture was defined as when the colonoscope could not pass through an anastomosis due to intraluminal narrowing. In these cases, the absence of can- 
cer recurrence was confirmed by biopsy. CV-290 colonoscopes were used in this study (Olympus Medical Systems Co. Ltd., Tokyo, Japan). Mechanical bowel preparation was accomplished using 4-L PEG (CoLyte; Taejoon Pharm. Co.) or 2-L sodium picosulfate/magnesium citrate (Picolight, Pharmbio, Seoul, Korea). Choice of bowel preparation between conventional-volume (CoLyte) and low-volume (Picolight) methods was determined by the surgeon. A highly trained, experienced nurse instructed all colonoscopy patients on the proper methods for bowel preparation. Patients also watched a video made by the Gastroenterology Center at our hospital that was specifically produced to explain proper bowel preparation. The withdrawal time of patients who underwent a colonoscopy averaged more than 6 minutes. A successful examination was defined as when the colonoscope reached the cecum. Confirmation of the cecum occurred when both the appendiceal orifice and the ileocecal valve were identified.

The Boston bowel preparation scale was used to assess bowel preparation quality of the right colon, transverse colon, and rectosigmoid colon. The grade of each segment was measured by the endoscopist and based on a scale 0 to 3 ( 0 being poor and 3 being excellent), defined as follows: 0 , unprepared colon segment with mucosa not visible due to solid stool that cannot be cleared; 1 , portion of the mucosa of the colon segment visible but other areas of the colon segment not as visible due to staining, residual stool, and/or opaque liquid; 2 , minor amount of residual staining, small fragments of stool and/or opaque liquid, but the mucosa of the colon segment is visible; and 3, entire mucosa of the colon segment visible with no residual staining, small fragments of stool, or opaque liquid [11].

\section{Statistical analysis}

Data were analyzed using STATA ver. 13 (StataCorp LP, College Station, TX, USA). Descriptive statistics were reported as mean \pm standard deviation and categorical variables were reported as a ratio. Differences between patient age and bowel preparation method were analyzed with independent t-tests. Pearson chisquare tests or logistic regressions were used for bivariate analyses based on data distributions. Multiple logistic regression models were used to assess the risk factors for IBP. A P-value $\leq 0.05$ was considered statistically significant.

\section{RESULTS}

\section{Patient characteristics}

Medical records from 1,393 patients with histologically proven colon cancer who were candidates for curative left colectomy at the Department of Surgery, Kangbuk Samsung Hospital between June 2013 and June 2016 were initially included in this study. Of these, 76 patients were excluded due to the possibility of decreased bowel motility (Table 1). In addition, 8 patients were excluded due to benign anastomotic stricture and 2 patients were excluded because they had been treated with a method different
Table 1. Exclusion criteria

\begin{tabular}{|c|c|}
\hline Exclusion criteria & No. of patients \\
\hline \multicolumn{2}{|l|}{ Operative factors } \\
\hline \multicolumn{2}{|l|}{ With colorectal resection } \\
\hline $\begin{array}{l}\text { Liver resection including hemi-hepatectomy, tumorectomy, } \\
\text { segmentectomy }\end{array}$ & 15 \\
\hline Total abdominal hysterectomy or myomectomy & 6 \\
\hline Multiple colorectal resection & 6 \\
\hline (Partial) bladder resection or repair & 5 \\
\hline Small bowel resection and anastomosis & 4 \\
\hline Left nephrectomy & 3 \\
\hline Open conversion & 3 \\
\hline Subtotal gastrectomy & 2 \\
\hline \multicolumn{2}{|l|}{ After colorectal resection } \\
\hline Incisional herniorrhaphy & 6 \\
\hline Adhesiolysis & 6 \\
\hline lleostomy formation due to anastomosis leakage & 4 \\
\hline Retroperitoneal mass excision & 3 \\
\hline Presacral mass excision & 2 \\
\hline Internal iliac mass excision & 1 \\
\hline Liver tumorectomy & 1 \\
\hline \multicolumn{2}{|l|}{ Luminal factors } \\
\hline Benign stricture & 8 \\
\hline Antiperistaltic side-to-side anastomosis & 2 \\
\hline Obstruction due to disease progression & 1 \\
\hline Total & 76 \\
\hline
\end{tabular}

than end-to-end anastomosis. Of the 1,317 patients included in the final sample for the statistical analysis, 1,041 (79\%) were in the adequate preparation group and $286(21 \%)$ were in the IBP group. Mean age of patients at the time of colonoscopy for the adequate bowel preparation group was $65.4 \pm 11.3$ years and for the IBP group was $65.2 \pm 11.4$ years. With regard to left colectomy, $52.2 \%$ of patients underwent low anterior resection, $43.9 \%$ underwent anterior resection, and 3.9\% underwent left hemicolectomy (Table 2). Mean patient body mass index (BMI) was $26.4 \pm 24.9 \mathrm{~kg} / \mathrm{m}^{2}$. A total of 121 patients $(9.2 \%)$ were identified as having diabetes mellitus. The sex ratio of patients was skewed toward males (64.7\% vs. $35.3 \%$ ). A total of 324 patients (24.6\%) underwent systemic treatment. Three times more patients underwent a colonoscopy after taking low-volume bowel preparation drugs compared to those that took conventional-volume drugs (75.4\% vs. $24.6 \%)$. Approximately 20\% of patients underwent a surveillance colonoscopy within 1 year after colorectal resection.

Table 3 shows the results of both univariate and multivariable logistic regression analyses for identifying risk factors associated with IBP. In the multivariable analysis, after controlling for patient 
Annals of Bowel Preparation for Surveillance Colonoscopy After Colorectal Resection: A New Perspective

Coloproctology Donghyoun Lee and Ho-Kyung Chun

Table 2. Baseline characteristics and association of potential factors with bowel preparation

\begin{tabular}{|c|c|c|c|c|}
\hline Characteristic & Total $(n=1,317)$ & Adequate $(n=1,041,79 \%)$ & Inadequate $(\mathrm{n}=286,21 \%)$ & P-value \\
\hline \multicolumn{5}{|l|}{ Operation type } \\
\hline Low anterior resection & $688(52.2)$ & $552(79.9)$ & $139(22.1)$ & 0.17 \\
\hline Left hemicolectomy & $51(3.9)$ & $46(80.7)$ & $11(21.6)$ & \\
\hline Age (yr) & $65.6 \pm 11.3$ & $65.4 \pm 11.3$ & $65.2 \pm 11.4$ & \\
\hline $70-80$ & $353(26.8)$ & $274(77.6)$ & 79 (22.4) & 0.95 \\
\hline$>80$ & $129(9.8)$ & $90(69.8)$ & 39 (30.2) & 0.02 \\
\hline \multicolumn{5}{|l|}{ Sex } \\
\hline Male & $852(64.7)$ & $674(78.5)$ & $185(21.5)$ & 0.73 \\
\hline Overweight (23-25) & $330(25.1)$ & $261(79.1)$ & $69(20.9)$ & 0.85 \\
\hline Obese (>25) & $364(27.6)$ & $273(75.0)$ & $91(25.0)$ & 0.06 \\
\hline \multicolumn{5}{|l|}{$\mathrm{DM}$} \\
\hline Non-DM & $1,196(90.8)$ & $993(78.1)$ & $263(21.9)$ & 0.18 \\
\hline $\mathrm{DM}$ & $121(9.2)$ & $88(62.5)$ & $33(27.0)$ & \\
\hline \multicolumn{5}{|l|}{ Duration (yr) } \\
\hline$\leq 1$ & $256(19.4)$ & $180(69.2)$ & $76(30.8)$ & 0.00 \\
\hline $1-2$ & $252(19.1)$ & $194(76.9)$ & $58(23.1)$ & 0.01 \\
\hline $2-3$ & $271(20.7)$ & $222(81.9)$ & 49 (18.1) & 0.04 \\
\hline Systemic & $324(24.6)$ & $239(73.8)$ & 85 (26.2) & \\
\hline
\end{tabular}

Values are presented as the mean \pm standard deviation or number (\%).

DM, diabetes mellitus.

age, sex, BMI, timing of postsurgery colonoscopy, and bowel preparation drug, age $>80$ years (odds ratio [OR], 1.62 ; $95 \%$ confidence interval $[\mathrm{CI}], 1.08-2.42 ; \mathrm{P}=0.02)$ and a surveillance colonoscopy within one year after surgery (OR, 1.66; 95\% CI, 1.222.27; $\mathrm{P}=0.01$ ) were associated with a significantly increased risk of IBP. The proportion of IBP in the low-volume group was significantly higher than in the conventional-volume group within one year after colorectal resection (33.1\% vs. $22.2 \% ; \mathrm{P}=0.03$ ) (Table 4 ).

\section{DISCUSSION}

Many studies have examined the optimal interval for postcolorec- tal resection surveillance colonoscopy and bowel preparation for colonoscopy in the general population. However, no studies have assessed risk factors for IBP differentiating surveillance colonoscopy and other colonoscopies including preoperative or screening colonoscopy. In addition, no previous studies have focused on aspects of bowel preparation for surveillance colonoscopy. In this study, we measured IBP rates among patients who underwent colorectal resection due to CRC, and analyzed various factors affecting IBP postsurgery.

Widely-known causes of IBP include patient-related factors such as old age, comorbidity, poor compliance for bowel preparation, and prior history of abdominal surgery and endoscopy unit 
Table 3. Association of potential risk factors and inadequate bowel preparation

\begin{tabular}{|c|c|c|c|c|c|c|c|}
\hline \multirow{2}{*}{ Variable } & \multirow{2}{*}{ №. (\%) } & \multicolumn{3}{|c|}{ Unadjusted } & \multicolumn{3}{|c|}{ Adjusted } \\
\hline & & $\mathrm{OR}$ & $95 \% \mathrm{Cl}$ & P-value & $\mathrm{OR}$ & $95 \% \mathrm{Cl}$ & P-value \\
\hline \multicolumn{8}{|l|}{ Age (yr) } \\
\hline $60-70$ & $399(30.3)$ & 0.81 & $0.61-1.08$ & 0.16 & 0.80 & $0.98-1.07$ & 0.08 \\
\hline $70-80$ & $353(26.8)$ & 0.99 & $0.74-1.32$ & 0.28 & 1.18 & $0.86-1.62$ & 0.20 \\
\hline Male sex & $852(64.7)$ & 0.95 & $0.71-1.27$ & 0.73 & 0.99 & $0.74-1.33$ & 0.00 \\
\hline \multicolumn{8}{|l|}{ Body mass index $\left(\mathrm{kg} / \mathrm{m}^{2}\right)$} \\
\hline Normal (<23) & $623(47.3)$ & 0.93 & $0.71-1.20$ & 0.59 & 1.10 & $0.73-1.64$ & 0.28 \\
\hline Overweight (23-25) & $330(25.1)$ & 0.88 & $0.65-1.19$ & 0.42 & 0.86 & $0.63-1.17$ & 0.23 \\
\hline Obese (>25) & $364(27.6)$ & 1.21 & $0.91-1.61$ & 0.17 & 1.23 & $0.77-1.96$ & 0.23 \\
\hline $1-2$ & $252(19.1)$ & 0.63 & $0.43-0.93$ & 0.01 & 1.01 & $0.73-1.40$ & 0.30 \\
\hline $2-3$ & $271(20.7)$ & 0.69 & $0.49-0.99$ & 0.03 & 0.72 & $0.51-1.01$ & 0.02 \\
\hline$\geq 3$ & $538(40.9)$ & 0.81 & $0.57-1.12$ & 0.20 & 0.85 & $0.65-1.11$ & 0.05 \\
\hline Low-volume preparation & $993(75.3)$ & 1.33 & $0.97-1.83$ & 0.23 & 1.37 & $1.00-1.88$ & 0.27 \\
\hline Systemic therapy & 324 (24.6) & 1.24 & $0.95-1.61$ & 0.10 & 1.21 & $0.93-1.59$ & 0.00 \\
\hline
\end{tabular}

OR, odds ratio; $\mathrm{Cl}$, confidence interval.

Table 4. Proportion of inadequate bowel preparation (IBP) by bowel preparation methods

\begin{tabular}{llll}
\hline Duration between surgery and colonoscopy & IBP rate & P-value \\
\hline$\leq 1$ Year & Total $(n=256)$ & $29.7(n=76)$ & \\
& Conventional $(n=81)$ & $22.2(n=18)$ & 0.03 \\
& Low $(n=175)$ & $33.1(n=58)$ & \\
1-2 Years & Total $(n=252)$ & $27.0(n=68)$ & \\
& Conventional $(n=44)$ & $18.2(n=8)$ & 0.59 \\
2-3 Years & Low $(n=208)$ & $28.8(n=60)$ & \\
& Total $(n=272)$ & $18.0(n=49)$ & \\
& Conventional $(n=80)$ & $16.3(n=13)$ & 0.07 \\
$>3$ Years & Low $(n=192)$ & $18.8(n=36)$ & \\
& Total $(n=537)$ & $21.0(n=113)$ & \\
& Conventional $(n=117)$ & $18.8(n=22)$ & 0.63 \\
\hline & Low $(n=420)$ & $21.7(n=91)$ & \\
\hline
\end{tabular}

factors including long wait times for colonoscopy after bowel preparation [12]. Previous studies reported the proportion of IBP was approximately $15 \%-25 \%$ in the general population [9], and similar results were obtained in our study population. Interestingly, we observed a significant increase in IBP rate during surveillance colonoscopy within 1 year after colorectal resection compared to most other studies, which reported approximately $15 \%-25 \%$ of patients with IBP who did not have a history of abdominal surgery [5].

We hypothesized that the discrepancy between this study and past studies is due to bowel resection or denervation, both of which affect colon motility. The effects manifest as low anterior syndrome (LARS) or anterior resection syndrome (ARS), which include a wide range of symptoms including fecal incontinence, urgency or frequency of stools, and constipation. Previous studies have shown that greater than $90 \%$ of patients suffer from symptoms of bowel function change after low anterior resection [13]. Among LARS and ARS patients, 47\% experienced evacuatory dysfunction [14]. We assumed that these symptoms were associated with IBP after colorectal resection. Specifically, existing studies reported that severe constipation can occur due to denervation after division of the lateral ligaments of the rectum [15]. Experiments using dogs showed that denervation of the presacral pelvic plexus led to continued decrease of colonic motility for at least 6-month postoperation [16]. Another study using a guinea pig model reported that 57-60 days were required for bowel motility recovery by checking phase III migration and immunoreactivities such as vasoactive intestinal peptide, gastrin-releasing peptide, and somatostatin in nerve fibers after bowel anastomosis [16]. Although little research has been conducted to investigate the relationship between bowel motility and colorectal resection in hu- 
mans, decreased colon motility may be one of the reasons for increased IBP rate after colorectal resection [17]. In addition, our study suggests that the highest IBP rate within 1 year of surgery and its subsequent decline is consistent with previous studies that documented an improvement in symptoms associated with LARS or ARS over a period of 1 to 2 years [18].

Poor patient compliance is often used to explain IBP [19]. The most commonly cited cause of poor compliance is patient unwillingness to ingest a large volume of bowel preparation drug. It has been reported that the IBP percent due to failure to consume the entire $4 \mathrm{~L}$ of PEG is about 5\%-15\% [20]. Recent studies reported that two sachets of oral sodium PICO were as effective as $236 \mathrm{~g}$ oral PEG as a bowel preparation drug for the general population and substituting low-volume PICO for conventional-volume PEG may decrease IBP rates. Based on these results, the use of low-volume drugs has become increasingly common among many hospitals in South Korea, including ours. However, no study has shown that low-volume drugs are as effective for surveillance colonoscopy. We observed a significantly high IBP rate in the lowvolume group during surveillance colonoscopy, especially within 1 year after surgery. To the best of our knowledge, this is the first study that investigated both IBP rates over time after colorectal resection and the effects of the low-volume drug for surveillance colonoscopy.

Previous studies considered age as a factor that increased the risk of IBP [21, 22]. IBP rates for colonoscopies in the very elderly were reported to range from $12.1 \%$ to $25.9 \%$ [21, 23]. This result is broadly consistent with previous studies documenting that slower colonic transit and increased incidence of obstipation may be the cause of IBP among elderly patients [22, 24]. However, a recent large-scale prospective study showed that age was not a significant risk factor for IBP [25], and no study to date has evaluated age as a risk factor for IBP for surveillance colonoscopy. Other studies reported being 60-65 years of age and over as a risk factor for IBP. However, our multivariate analysis showed that being over 80 years of age is an independent risk factor of IBP after colorectal resection. One possible explanation was that our study included patients that underwent CRC surgery and received regular follow-up checks in our hospital. Therefore, our study patients could have relatively better compliance rates than patients treated in other hospitals.

Systemic therapy could affect bowel preparation. Constipation is the third most common symptom among patients receiving cytotoxic chemotherapy, with an overall prevalence of $16 \%$ (with 5\% classified as severe and $11 \%$ classified as moderate) [26, 27]. Prior studies have shown that chemotherapy affects the gastro-intestinal tract nerve endings, causing changes in motor activity [27]. No large-scale prospective study that examined bowel preparation quality in patients who underwent systemic treatment has been conducted; however, our study shows that systemic treatment is significantly associated with IBP.

Several studies have evaluated male sex as a risk factor for IBP
$[25,28]$. According to a recent study conducted in the United States and Malaysia, males are 1.4-1.6 times more likely to have a risk of IBP $[12,28]$. This difference is presumably because males are less likely to pursue disease prevention and are less healthconscious than females [29]. Contrary to existing studies, our findings indicate absence of gender differences in IBP rates. In addition, male gender was not a statistically significant independent risk factor in multiple logistic regression. We believe that this result is probably due to our sample characteristics, as our sample consisted of patients who underwent CRC surgery and subsequent follow-ups indicating that they were likely to be relatively more health-conscious or better compliant in bowel preparation.

We acknowledge several limitations associated with this study. Patients studied were from a single center and the retrospective nature of the study can lead to selection bias. This study reports that colorectal resection could affect IBP, but we were not able to examine the relationship between IBP and its long-term impacts, including disease-free and/or progression-free survival. This hypothesis and conclusion should be validated by prospective multicenter trials, allowing us to analyze results from consecutive postcolorectal resection surveillance colonoscopies and study how IBP for surveillance colonoscopy affects long-term prognosis in CRC patients. Another limitation to this study is that we only compared patients who underwent left colectomy with end-toend anastomosis. Patients who underwent other colorectal resections, such as the Hartmann's procedure, abdominoperineal resection, and right hemicolectomy were excluded to maintain homogeneity among patients included in this study. Due to this limitation, it was difficult to conclude that our findings apply to patients who had other colon cancer surgeries. Finally, another limitation of this study involves the issue of anastomotic level. The level of anastomosis is an important factor in the cause of LARS. In this study, we distinguished anterior resection/low anterior resection based on peritoneal reflection [30]. However, the number of sphincter-saving surgeries such as ultra-low anterior resection or intersphincteric resection has increased recently due to the development of neoadjuvant therapy. Therefore, we suggest that further research be conducted to compare bowel motility and bowel preparation after colorectal resection according to more detailed classification by anastomotic height.

Little is known about changes in bowel motility and the enteric nervous system after CRC resection. Despite these limitations, our results have important implications for surveillance colonoscopy after colorectal resection. Our current investigation showed increased IBP rates for surveillance colonoscopy, which was highest within one year after colorectal resection. In addition, the use of low-volume bowel preparation after colorectal resection should be sufficiently reconsidered because of the IBP impacts. To conclude, we suggest an increase in bowel preparation drug volume or changes in drug combinations and adjustments in time between bowel preparation and a colonoscopy for surveillance colonoscopy. Furthermore, low-volume preparation drugs should be 
avoided for surveillance colonoscopy conducted within 1 year of surgery.

\section{CONFLICT OF INTEREST}

No potential conflict of interest relevant to this article was reported.

\section{REFERENCES}

1. Kim DH. Colorectal cancer epidemic in Korea. Ann Coloproctol 2013;29:1-4.

2. Arnold M, Sierra MS, Laversanne M, Soerjomataram I, Jemal A, Bray F. Global patterns and trends in colorectal cancer incidence and mortality. Gut 2017;66:683-91.

3. Kahi CJ, Boland CR, Dominitz JA, Giardiello FM, Johnson DA, Kaltenbach T, et al. Colonoscopy surveillance after colorectal cancer resection: recommendations of the US multi-society task force on colorectal cancer. Gastrointest Endosc 2016;83:489-98. e10.

4. le Clercq CM, Winkens B, Bakker CM, Keulen ET, Beets GL, Masclee AA, et al. Metachronous colorectal cancers result from missed lesions and non-compliance with surveillance. Gastrointest Endosc 2015;82:325-33.e2.

5. Kahi CJ, Boland CR, Dominitz JA, Giardiello FM, Johnson DA, Kaltenbach T, et al. Colonoscopy surveillance after colorectal cancer resection: recommendations of the US Multi-Society Task Force on Colorectal Cancer. Gastroenterology 2016;150:758-68. e11.

6. Benson AB 3rd, Venook AP, Cederquist L, Chan E, Chen YJ, Cooper HS, et al. Colon cancer, version 1.2017, NCCN clinical practice guidelines in oncology. J Natl Compr Canc Netw 2017; 15:370-98.

7. Rex DK, Petrini JL, Baron TH, Chak A, Cohen J, Deal SE, et al. Quality indicators for colonoscopy. Am J Gastroenterol 2006;101: 873-85.

8. Chokshi RV, Hovis CE, Hollander T, Early DS, Wang JS. Prevalence of missed adenomas in patients with inadequate bowel preparation on screening colonoscopy. Gastrointest Endosc 2012; 75:1197-203.

9. Harewood GC, Sharma VK, de Garmo P. Impact of colonoscopy preparation quality on detection of suspected colonic neoplasia. Gastrointest Endosc 2003;58:76-9.

10. Fortin CN, Saed GM, Diamond MP. Predisposing factors to postoperative adhesion development. Hum Reprod Update 2015;21: 536-51.

11. Lai EJ, Calderwood AH, Doros G, Fix OK, Jacobson BC. The Boston bowel preparation scale: a valid and reliable instrument for colonoscopy-oriented research. Gastrointest Endosc 2009;69(3 Pt 2):620-5.

12. Chan WK, Saravanan A, Manikam J, Goh KL, Mahadeva S. Appointment waiting times and education level influence the quality of bowel preparation in adult patients undergoing colonoscopy. BMC Gastroenterol 2011;11:86.

13. Dennett ER, Parry BR. Misconceptions about the colonic Jpouch: what the accumulating data show. Dis Colon Rectum 1999;42:804-11.

14. Keane C, Wells C, O'Grady G, Bissett IP. Defining low anterior resection syndrome: a systematic review of the literature. Colorectal Dis 2017;19:713-22.

15. Speakman CT, Madden MV, Nicholls RJ, Kamm MA. Lateral ligament division during rectopexy causes constipation but prevents recurrence: results of a prospective randomized study. $\mathrm{Br} J$ Surg 1991;78:1431-3.

16. Ishikawa M, Mibu R, Iwamoto T, Konomi H, Oohata Y, Tanaka M. Change in colonic motility after extrinsic autonomic denervation in dogs. Dig Dis Sci 1997;42:1950-6.

17. Cooper GS, Chak A, Koroukian S. The polyp detection rate of colonoscopy: a national study of Medicare beneficiaries. Am J Med 2005;118:1413.

18. Pedersen IK, Christiansen J, Hint K, Jensen P, Olsen J, Mortensen $\mathrm{PE}$. Anorectal function after low anterior resection for carcinoma. Ann Surg 1986;204:133-5.

19. Kolts BE, Lyles WE, Achem SR, Burton L, Geller AJ, MacMath T. A comparison of the effectiveness and patient tolerance of oral sodium phosphate, castor oil, and standard electrolyte lavage for colonoscopy or sigmoidoscopy preparation. Am J Gastroenterol 1993;88:1218-23.

20. DiPalma JA, Marshall JB. Comparison of a new sulfate-free polyethylene glycol electrolyte lavage solution versus a standard solution for colonoscopy cleansing. Gastrointest Endosc 1990;36:2859.

21. Duncan JE, Sweeney WB, Trudel JL, Madoff RD, Mellgren AF. Colonoscopy in the elderly: low risk, low yield in asymptomatic patients. Dis Colon Rectum 2006;49:646-51.

22. McNabb-Baltar J, Dorreen A, Al Dhahab H, Fein M, Xiong X, O' Byrne $\mathrm{M}$, et al. Age is the only predictor of poor bowel preparation in the hospitalized patient. Can J Gastroenterol Hepatol 2016;2016:2139264.

23. Tsutsumi S, Fukushima H, Osaki K, Kuwano H. Feasibility of colonoscopy in patients 80 years of age and older. Hepatogastroenterology 2007;54:1959-61.

24. Loffeld RJ, Liberov B, Dekkers PE. Yearly diagnostic yield of colonoscopy in patients age 80 years or older, with a special interest in colorectal cancer. Geriatr Gerontol Int 2012;12:298-303.

25. Ness RM, Manam R, Hoen H, Chalasani N. Predictors of inadequate bowel preparation for colonoscopy. Am J Gastroenterol 2001;96:1797-802.

26. Yamagishi A, Morita T, Miyashita M, Kimura F. Symptom prevalence and longitudinal follow-up in cancer outpatients receiving chemotherapy. J Pain Symptom Manage 2009;37:823-30.

27. McQuade RM, Stojanovska V, Abalo R, Bornstein JC, Nurgali K. Chemotherapy-induced constipation and diarrhea: pathophysiology, current and emerging treatments. Front Pharmacol 2016; 


\section{Coloproctology Donghyoun Lee and Ho-Kyung Chun}

7:414.

28. Lebwohl B, Wang TC, Neugut AI. Socioeconomic and other predictors of colonoscopy preparation quality. Dig Dis Sci 2010;55: 2014-20.

29. Green CA, Pope CR. Gender, psychosocial factors and the use of medical services: a longitudinal analysis. Soc Sci Med 1999;48: 1363-72.

30. Ho YH. Techniques for restoring bowel continuity and function after rectal cancer surgery. World J Gastroenterol 2006;12:625260. 